\title{
Victimes « au carré » : dénonciation des iniquités dans la reconnaissance des victimes d'attentats (France, 2015)
}

"Squared" Victims: Denouncing Inequities in the Recognition of Terrorist Attacks' Victims (France, 2015)

\section{Marianne Doury}

\section{(2) OpenEdition Journals}

\section{Édition électronique}

URL : http://journals.openedition.org/aad/3452

DOI : 10.4000/aad.3452

ISSN : 1565-8961

\section{Éditeur}

Université de Tel-Aviv

\section{Référence électronique}

Marianne Doury, «Victimes « au carré » : dénonciation des iniquités dans la reconnaissance des victimes d'attentats (France, 2015)", Argumentation et Analyse du Discours [En ligne], 23 | 2019, mis en ligne le 18 octobre 2019, consulté le 28 novembre 2019. URL : http://journals.openedition.org/aad/ 3452 ; DOl : 10.4000/aad.3452

Ce document a été généré automatiquement le 28 novembre 2019.

\section{(c) $(1) \subseteq$}

Argumentation \& analyse du discours est mis à disposition selon les termes de la licence Creative Commons Attribution - Pas d'Utilisation Commerciale - Pas de Modification 4.0 International. 


\title{
Victimes « au carré » : dénonciation des iniquités dans la reconnaissance des victimes d'attentats (France, 2015)
}

\author{
"Squared" Victims: Denouncing Inequities in the Recognition of Terrorist \\ Attacks' Victims (France, 2015)
}

\section{Marianne Doury}

1 La psychologie sociale a élaboré le concept de « croyance en un monde juste » (Belief in a Just World), biais cognitif qui consiste à penser qu'on mérite ce qu'on obtient - ou qu'on obtient ce qu'on mérite : «The just world hypothesis states that people have a need to believe that their environment is a just and orderly place where people usually get what they deserve» (Lerner \& Miller 1978: 1030). Cette croyance, poursuivent les auteurs, permet aux individus de faire face à leur environnement physique et social comme s'il était stable et ordonné. Sans une telle croyance, il leur serait difficile de s'engager dans quelque action à long terme que ce soit, ou même de conformer leur comportement aux normes qui gouvernent la vie sociale au quotidien. Cette croyance en un monde juste est brutalement mise en cause lorsqu'adviennent des événements qui causent des préjudices graves à des individus, sans qu'il soit possible d'établir un lien entre le comportement de ces derniers et ce qui leur arrive. Diverses stratégies sont alors mises en œuvre afin de préserver la croyance en un monde juste face à ce qui pourrait être vu comme son invalidation catégorique (Lerner \& Simmons 1966, Lerner \& Miller 1978, Hafer \& Gosse 2011, Soudan \& Gangloff 2012, Corey et al. 2015).

2 Un attentat est, par excellence, un de ces événements susceptibles de mettre à mal la croyance que le monde est juste, en particulier lorsque les victimes sont catégorisées comme « innocentes ». Dans le cas des attaques terroristes islamistes qui ont eu lieu en 2015 sur le sol français, l'ébranlement de la croyance en un monde juste du fait des attentats eux-mêmes s'est, pour certains, doublé d'un ébranlement lié au traitement réservé aux victimes par les médias. En effet, parmi les réactions qui ont suivi les 
attaques, de nombreux commentaires postés sur Internet critiquent le traitement des victimes par les médias ou les politiques, et dénoncent une déploration jugée inéquitable de différentes catégories de victimes. On s'interrogera ici sur ces dénonciations d'un «deux poids, deux mesures", qui ferait des victimes moins reconnues, moins « légitimes » (Baudinière 2008), et partant, moins médiatisées, des victimes « au carré », atteintes dans leur chair par la violence terroriste, atteintes une seconde fois par une prise en considération jugée désinvolte.

Dans cette perspective, après avoir spécifié l'approche de l'argumentation mobilisée, on exploitera les réflexions de Perelman sur la règle de justice. On montrera comment le non-respect de la règle de justice en matière de reconnaissance publique des victimes peut servir deux lignes argumentatives bien distinctes. L'une - sous-représentée dans les commentaires - est au bénéfice desdites victimes, et vise à les rétablir dans leurs droits (symboliques) : il s'agirait de rétablir la justice, et de traiter équitablement toutes les victimes d'attentat. L'autre, massivement présente, fait de la dénonciation d'une injustice un point de départ vers une critique sociale plus ou moins articulée, et ouvre un espace d'expression du ressentiment, dont on mettra en évidence les diverses déclinaisons. On conclura sur ce que de telles critiques suggèrent, en miroir, du sentiment d'injustice subie ressenti par ceux qui les profèrent.

\section{Quelle approche de l'argumentation?}

\subsection{Une double visée de positionnement et de justification}

4 On abordera ici l'argumentation comme une activité communicationnelle située (ancrée dans une situation d'énonciation dont il est impossible de faire abstraction), mise en œuvre dans des discours en interaction, au sens propre (dans le cas d'une confrontation dialogale entre des interlocuteurs défendant des positions divergentes) comme au sens figuré (dans le cas d'un discours monolocuteur faisant la place au contre-discours grâce à un feuilletage énonciatif plus ou moins complexe). L'argumentation sera traitée dans une perspective descriptive: il s'agit de rendre compte de la façon dont des locuteurs s'y prennent lorsqu'ils cherchent à défendre une position en situation de dissensus, potentiel ou effectif. Le cœur de l'argumentation est la mise en œuvre d'une sorte de mécanique discursive qui lie l'expression d'une position à l'horizon d'un contre-discours; elle est vue comme un mode de construction du discours visant à le rendre plus résistant à la contestation.

5 Jacquin et Micheli (2012) ont clairement posé la double caractérisation de l'argumentation ainsi conçue, celle-ci poursuivant tout à la fois une visée de positionnement (par laquelle un locuteur "prend position " sur une question) et une visée de justification (cette position doit être construite, adossée à des étais langagiers qui en contrarient la contestation).

6 Chacune de ces visées détermine ce que Micheli (2012) appelle des «corrélats langagiers ", des phénomènes linguistiques et discursifs spécifiques qui en permettent la réalisation. La visée de positionnement invite l'analyste à s'intéresser aux modes d'articulation du discours et du contre-discours (puisque prendre position sur un sujet, c'est d'une certaine façon "se poser en s'opposant ", s'affirmer par différenciation), aux manifestations de l'hétérogénéité énonciative, c'est-à-dire à tous les mécanismes discursifs qui permettent, dans un discours, de faire entendre d'autres « voix », d'autres 
points de vue, par rapport auxquels on se situe, auxquels on s'identifie ou dont on se distancie. La visée de justification, quant à elle, oriente l'attention vers la séquence argumentative (composée prototypiquement d'au moins un énoncé-argument et un énoncé-conclusion); elle demande qu'on s'interroge sur le principe de cohérence d'une telle séquence, et qu'on recoure à une linguistique textuelle ou transphrastique; ce sont des interrogations sur la visée justificative de l'argumentation qui peuvent amener à s'intéresser aux indicateurs argumentatifs - notamment, mais pas seulement, aux fameux « connecteurs argumentatifs » ou « connecteurs logiques »-, et, ce qui va nous intéresser à présent, aux types d'arguments.

\subsection{Les types d'arguments}

7 Si multiples que soient les cadres disciplinaires de référence ou les positionnements théoriques qui orientent les recherches, la plupart des chercheurs en argumentation se retrouvent autour de quelques catégories transversales qui constituent le terreau commun des réflexions. La notion de type d'argument compte parmi celles-là.

8 La notion de «type d'argument » repose sur l'idée qu'on peut regrouper les différents arguments en fonction de la nature de la relation qui permet le passage de la prémisseargument à la conclusion - argumentations comparatives, parmi lesquelles on trouve l'argumentation par le précédent, l'argument a fortiori, l'argument a pari; argumentations causales, parmi lesquelles, l'argument pragmatique ou l'argument de direction; argumentations symptomatiques, dont l'argument d'autorité... ${ }^{1}$. Les types d'arguments constituent une catégorie d'analyse particulièrement précieuse en ce qu'elle permet un "décollage" du contenu littéral et spécifique d'un discours argumenté pour opérer une forme de montée en généralité qui autorise des rapprochements entre différents discours argumentés présentant des contenus divers, mais mobilisant des structures argumentatives similaires².

9 Venons-en à présent non pas à un type d'argument spécifique, mais à un ensemble arguments relevant de types différents, mais qui ont pour caractéristique commune de faire appel à ce que Chaïm Perelman désigne par « règle de justice ».

\section{Argumentation et règle de justice}

\subsection{La règle de justice}

10 Chaïm Perelman, en tant que philosophe du droit, a livré une riche réflexion sur la notion de justice, et l'a articulée à des structures argumentatives spécifiques ${ }^{3}$. Perelman s'interroge sur la définition du mot « justice », dont il souligne le sémantisme confus et émotionnellement chargé. Il s'agit, dit-il, d'une de ces notions à propos desquelles on ne peut tomber d'accord sur un cœur conceptuel indiscutable, et que, même de bonne foi, chacun définit de telle sorte que lui seul puisse s'en revendiquer et en exclure l'adversaire.

11 Il énonce ainsi quelques-uns des principes dont peuvent se réclamer ceux qui affirment « dire » la justice (1990 [1945] : 19-23):

1. A chacun la même chose

2. A chacun selon ses mérites

3. A chacun selon ses œuvres 
4. A chacun selon ses besoins

5. A chacun selon son rang

6. A chacun selon ce que la loi lui attribue

12 Ces principes sont, selon Perelman, inconciliables, et on comprend bien pourquoi : dans des situations concrètes, le recours à l'un ou l'autre conduira à des jugements très différents.

Perelman ne s'en tient pas à ce constat d'irréductibilité ; il s'interroge sur ce à quoi pourrait ressembler une notion commune qui constituerait une définition de la justice «formelle » ou "abstraite ", dont chaque formule particulière ou " concrète » de la justice (principes de 1 à 6) constituerait une des innombrables instanciations. La formule générale, proposant une définition de la justice formelle ou abstraite, doit " contenir un élément indéterminé, ce qu'on appelle en mathématiques une variable, dont la détermination donnera tantôt l'une tantôt l'autre conception de la justice » (29) ; il propose alors de définir la justice formelle comme « un principe d'action selon lequel les êtres d'une même catégorie essentielle doivent être traités de la même façon » (30). Cette définition ne nous dit

ni quand deux êtres font partie d'une catégorie essentielle ni comment il faut les traiter. Nous savons qu'il faut traiter ces êtres non pas de telle ou telle façon, mais de façon égale, de sorte que l'on ne puisse pas dire que l'on a défavorisé l'un par rapport à l'autre. Nous savons aussi qu'un traitement égal ne doit être réservé qu'aux êtres faisant partie d'une même catégorie essentielle (31).

C'est ce principe général qui est supposé présider à tout jugement dans le domaine judiciaire et auquel font appel, on le verra, bien des argumentations au-delà de ce cadre spécifique.

Perelman identifie ensuite les angles sous lesquels un jugement peut être contesté.

Un jugement pourrait être qualifié d'injuste notamment :

$1^{\circ}$ parce qu'il applique une formule de la justice concrète que l'on n'admet pas (on défend le principe "à chacun selon ses besoins » alors que l'adversaire applique un " à chacun selon ses moyens ", ou « à chacun la même chose ») ;

$2^{\circ}$ parce qu'il conçoit la même formule de façon différente ;

$3^{\circ}$ parce que, à sa base, se trouve une représentation inadéquate des faits ;

$4^{\circ}$ parce qu'il enfreint les prescriptions de justice formelle demandant que l'on traite de la même façon les êtres faisant partie d'une même catégorie essentielle (34-35).

\subsection{Exploitation argumentative de la règle de justice}

Dans une perspective plus large d'analyse argumentative (et pas seulement d'argumentation judiciaire), la règle de justice, qui veut que l'on traite de la même façon les individus relevant d'une même catégorie, sous-tend bon nombre d'argumentations, qui ont toutes comme trait commun d'être orientées vers des thèses normatives, c'est-à-dire des conclusions qui réalisent des actes de langage comme un conseil, une injonction, une interdiction, une préconisation... et non des conclusions épistémiques, visant à « dire le vrai ». 


\subsubsection{Argument a pari et appel au précédent}

17 Reprenons un exemple de Plantin, et imaginons un père de famille qui, à sa fille cadette, âgée de 14 ans et désirant plus que tout au monde participer à une fête entre amis, dirait :

Tu ne sortiras pas ce soir ! Ta sœur a bien attendu 16 ans...

Ce faisant, il adosse une conclusion normative («tu ne sortiras pas ce soir!») à un argument («ta sœur a bien attendu 16 ans»), qui n'est pertinent vis-à-vis de la conclusion qu'en association avec la règle de justice, ainsi spécifiée : «Les membres d'une même fratrie doivent être traités de façon similaire ». Cette argumentation est caractéristique des raisonnements a pari, dont Perelman et Olbrechts-Tyteca (2013) posent la centralité dans la tradition juridique, puisqu'ils sont au fondement de l'appel au précédent.

19 L'orientation de l'argumentation faisant appel à la règle de justice peut être, hors contexte, indéterminée. En effet, lorsqu'elle est mobilisée dans une situation où on constate que deux cas A et B relevant d'une même catégorie n'ont pas été traités de façon similaire, et qu'on veut "rétablir la justice» en leur appliquant le même traitement, veut-on étendre le traitement appliqué à $\mathrm{A}$ jusqu'à $\mathrm{B}$, ou au contraire, appliquer à $\mathrm{A}$ le traitement dont $\mathrm{B}$ a fait l'objet ?

Il est cependant rare que celui qui a mis le doigt sur l'existence de traitements dissimilaires de deux cas relevant d'une même catégorie, et qui a demandé qu'il soit remédié à cette inégalité de traitement, n'ait pas lui-même une préférence sur la direction d'ajustement souhaitable. Considérons par exemple qu'un enfant, ayant jugé que sa copie a fait l'objet d'une notation injuste, aille voir son enseignant pour crier à l'injustice, en arguant du fait que son camarade, qui a répondu la même chose que lui, a obtenu deux points de plus. Cet enfant-là serait probablement bien désappointé si la solution proposée par l'enseignant était de baisser de deux points la note de son camarade - sauf si son sens de la justice est à ce point aiguisé que l'apaisement apporté par la restauration d'un traitement équitable l'emporte sur le fait que cette restauration a entraîné la sanction de son camarade.

Plus généralement, les orientations argumentatives concurrentes d'un appel à la règle de justice sont en réalité engagées dans des débats distincts. Ainsi, un argument fréquemment invoqué dans le cadre des discussions sur la dépénalisation du cannabis dit qu'il faut dépénaliser le cannabis, parce que la consommation d'alcool n'est pas sanctionnée légalement; or, cannabis et alcool peuvent être considérés comme relevant d'une même catégorie (celle des "drogues douces»). Certains opposants à la dépénalisation du cannabis sont prêts à entendre l'argument, mais à en tirer une conclusion que les partisans de cette mesure ne visaient pas: "il faut sanctionner pénalement la consommation d'alcool »; même catégorisation commune, même règle de justice, mais orientations argumentatives différentes. Dans ce dernier cas, il s'agit d'une forme de détournement, les mêmes pièces étant utilisées pour la reconstitution d'un tout autre puzzle argumentatif. On quitte le débat sur la légalisation du cannabis, pour entrer dans le débat sur la pénalisation de l'alcool. 


\subsubsection{Argument a contrario} " traitent de l'application ou de la non-application, à une autre espèce du même genre, de ce qui a été affirmé pour une espèce particulière » (Perelman et Olbrechts-Tyteca 1988 : 325). Il consiste à exploiter le principe selon lequel, si les éléments d'une même espèce doivent être traités de façon similaire, alors, a contrario, les membres de deux espèces distinctes d'un même genre doivent faire l'objet de traitements différenciés. Perelman prend l'exemple d'une loi qui régirait l'héritage des descendants mâles; un argument $a$ pari cherchera à étendre cette loi de sorte qu'elle s'applique également aux filles, sur la base de l'appartenance à un genre commun ("les descendants»); un argument a contrario s'opposera à une telle extension, considérant qu'au-delà de l'espèce initialement visée (les descendants mâles), le traitement ne s'applique pas.

\subsubsection{Argument a pari et argumentations comparatives}

Arguments a pari et argumentations comparatives sont parfois si proches qu'il est difficile de les distinguer, et Plantin signale qu' " on utilise parfois l'étiquette "argument a comparatione" ou le vocabulaire de l'analogie pour désigner l'argumentation a pari " (2016: 21). Il me semble pourtant préférable d'éviter de les superposer trop rapidement. En effet, l'argument a pari s'appuie, on l'a vu, sur une opération de catégorisation : c'est parce que $A$ et $B$ relèvent de la même catégorie que le jugement appliqué à A doit être étendu à B. Or, deux éléments d'une même catégorie vont, de ce fait, avoir l'un avec l'autre une certaine ressemblance (puisque, du fait de l'appartenance à cette catégorie commune, ils vont partager un certain nombre de propriétés).

On peut toujours, partant du constat de ressemblances entre $\mathrm{A}$ et $\mathrm{B}$, remonter à une catégorie à laquelle A et B appartiendraient tous deux (et si cette catégorie n'existe pas, la créer) : Isabelle et Corinne se ressemblent. Elles sont toutes deux blondes, vives, grandes, minces et elles parlent le serbo-croate; elles font partie de la catégorie des «blondes-vives-grandes-minces-qui-parlent-le-serbo-croate». Mais on traitera le parallèle entre Isabelle et Corinne plus volontiers en termes de ressemblance que de catégorisation. En revanche, le dialogue fictif exploité par Plantin entre le père et sa fille cadette sera plus volontiers abordé en termes de catégorisation. Certes, la fille cadette et sa sœur partagent au moins une, voire deux propriétés susceptibles d'être pertinentes en situation (celle d'avoir les mêmes parents - et d'être de sexe féminin); mais on traitera plutôt la situation en disant que les jeunes filles appartiennent à la même fratrie (une même catégorie), qu'en affirmant qu'elles « se ressemblent ».

\subsubsection{Argument de réciprocité}

Koren montre que, dans la pensée de Perelman, l'argument de réciprocité s'appuie également sur la règle de justice, puisque

[s]a mise en oeuvre implique l'intrication d'un raisonnement formel et de considérations pratiques, «fondées dans la nature des choses » $(\mathrm{RH}: 298)$. La notion pratique de «réciprocité" a le principe de "symétrie» pour condition de possibilité (ER : 83). Celui-ci implique en logique formelle que si « les termes a et $b$, antécédent et conséquent d'une relation $\mathrm{R}$ » (ER : 82), peuvent être intervertis de sorte que $a b$ est entièrement assimilable à $b a$, les deux types de relation peuvent

Argumentation et Analyse du Discours, 23 | 2019 
être traités de façon absolument identique.

Appliquer ce principe à des situations socio-historiques concrètes, c'est souligner le fait que deux situations distinctes sont néanmoins le "pendant l'une de l'autre " (TA 297) et qu'il est légitime de ce fait de leur appliquer le même traitement (2019: 254).

De même, pour Plantin, un locuteur qui ferait la leçon à un interlocuteur goujat en lui disant: «je suis poli avec vous, alors soyez poli avec moi », mobilise l'argument de réciprocité ; ce faisant, il «se définit lui-même et définit son partenaire comme des membres d'une même catégorie, qui doivent être traités de la même manière, V. Règle de justice. » (2016:501).

\section{Etude de cas}

La raison pour laquelle mon attention s'est portée sur des mécanismes argumentatifs du type de ceux dont on vient d'avoir un aperçu est que, comme d'autres (Badouard 2016: 215, Gombin, Mariau et Villeneuve 2016: 2514), j'ai été frappée, dans les commentaires qui ont fait suite à l'attentat commis contre le magazine Charlie Hebdo le 7 janvier 2015, par la récurrence d'argumentations reposant sur la règle de justice, soit appelant à son application, soit dénonçant sa transgression. J'ai alors recueilli un corpus d'exemples de ce phénomène rassemblant :

- 200 messages postés, dans le mois qui a suivi les attentats de janvier 2015, sur le site du Salon Beige, qui se présente comme un «blog quotidien d'actualité animé par des catholiques laïcs ", et que Dominique Albertini et David Doucet, dans leur enquête intitulée La Fachosphère. Comment l'extrême droite remporte la bataille du Net, décrivent comme une des multiples formes de présence de l'extrême-droite sur Internet (Albertini et Doucet $2016: 165 \mathrm{sv}$.).

- 200 messages postés sur différents sites internet dans les jours qui ont suivi l'attentat, essentiellement sur le compte Facebook officiel de Charlie Hebdo.

\subsection{Le marquage langagier du « deux poids, deux mesures »}

Koren (2019), dans l'introduction du chapitre qu'elle y consacre, souligne que les infractions à la règle de justice passent par des dénonciations formulaires aisément repérables, et qui mériteraient d'être répertoriées parmi les indicateurs attachés à un type d'argument (Doury 2018, van Eemeren et al. 2007), tant elles orientent avec fiabilité vers la détection de critiques d'argumentations reposant sur la règle de justice. Dans les données recueillies, ces dénonciations passent essentiellement par les marqueurs suivants ${ }^{5}$ :

\section{- « Deux poids, deux mesures »}

Le marquage langagier à la fois le plus récurrent et le plus univoque accompagnant la dénonciation d'une violation de la règle de justice est, précisément, l'utilisation de l'expression «deux poids, deux mesures», que l'on retrouve dans les exemples suivants :

Bref, voilà une opportunité rare qui a été gaspillée. Et tout cela pourquoi ?

Pour signifier que l'antisémitisme est une cause nationale? Et témoigner du fait qu'il y a bel et bien un $\mathbf{2}$ poids $\mathbf{2}$ mesures pour une communauté ? [SB] 
Charlie Hebdo jamais inquiété pour ses caricatures haineuses, par contre MINUTE pour une photo de la gay pride non truquée en couverture, je crois $10000 €$ d'amende, deux poids deux mesures ! ! [SB]

Ce que je voudrais souligner, c'est cet éternel 2 poids 2 mesures validé par les médias, qui approuvent tacitement que les musulmans se marient uniquement religieusement en toute impunité et pas les autres, et ce en opposition aux lois de ladite république. [SB]

La présence de cette expression est telle qu'elle donne lieu, sur le site du Salon Beige, à un développement philologique savant sur la graphie correcte de «deux poids, deux mesures» :

Je suggère que l'on utilise correctement l'expression « deux poids de mesure » et non plus en répétant le chiffre deux, ce qui est dénué de sens. En effet l'expression renvoie à l'habitude de peser le kilo de pain en utilisant dans l'un des plateaux de la balance un " poids de mesure » de un kilo. Il y avait tromperie quand le boulanger utilisait deux "poids de mesure " dont l'un faisait moins d'un kilo, afin de flouer certains clients. Les clients trompés sur la quantité achetée étaient fondés dans ce cas à se plaindre que soient utilisés « deux poids de mesure ». Merci. [SB]

31 - On trouve, dans le même esprit, des expressions comme "à sens unique ", «à géométrie variable » ou l'adjectif « sélectif », qui dénoncent la non-réciprocité, ou un traitement inéquitable, comme dans les exemples qui suivent :

Voilà, juste pour rappeler les valeurs de ce grand humaniste (visiblement humanisme à géométrie variable).... [SB]

J'ai cru que, subitement (et logiquement), il défendait le droit à manifester des musulmans et, par ailleurs, de tous ceux qui ne supportent pas la vulgarité de Charlie Hebdo. Car, après tout, la liberté d'expression ne doit pas être seulement à sens unique. [SB]

LIBERTE D'EXPRESSION OUI MAIS POUR TOUS!!! pour rappel (MANIFESTATION GAZA + DIEUDONNE) NON A LA LIBERTE D’EXPRESSION SELECTIVE ! [FB]

\section{- Selon que vous serez...}

Dans la série des marqueurs univoques, on trouve encore des citations ou des variations autour de la « chute » des animaux malades de la peste, « selon que vous serez puissant ou misérable » :

En revanche pour les 11 civils tués victimes d'un missile ukrainien d'aujourd'hui, il n'y aura sans doute pas un mot de nos medias. « selon que vous serez puissants ou misérables »... [SB]

Mais là, on ne parle pas de «bourde » de Charlie Hebdo, et on a bien raison. Selon que vous serez de gauche ou chrétien, aurait pu chanter Sardou... [SB] ${ }^{6}$

Selon que vous serez Charlie ou anonyme [SB]

\section{- Figures de contraste}

Enfin, et de façon moins spécifique, la dénonciation du non-respect de la règle de justice fait souvent appel à des figures de contraste, visant à mettre en relief les divergences de traitement. Ces figures s'appuient souvent sur des constructions binaires, dont chaque composante est marquée par des reprises soulignant le parallélisme, et par des antonymes exprimant la différence de traitement, comme dans les exemples que voici ${ }^{7}$ :

Ce qui est étrange c'est que si on est de gauche et antiraciste on peut critiquer

l'islam en faisant des caricatures, mais si on est comme Houellebecq ou Zemmour 
on est considéré comme islamophobe, je ne comprends pas... Il faudrait savoir si on peut critiquer l'islam ou pas... [SB]

Nous ne sommes pas dans un monde de bisounours. Aujourd'hui, nos politiques sont Charlie et hier ils demandaient la disparition de Zemmour de nos médias......La liberté d'expression dépend du sens du vent.... [SB]

La France égarée, marginalisée, reléguée des quartiers n'était pas dans les rues de Paris dimanche dernier. Les jeunes qui en sont issus ne comprennent tout simplement pas que le droit à l'irrespect et à la provocation puisse être sacralisé à Charlie-Hebdo et diabolisé pour Dieudonné. Méfions-nous du «deux poids, deux mesures ", car c'est la matrice des violences de demain [SB]

Lu dans les Echos: «Avec ses dessins, Charlie Hebdo a pu choquer certaines sensibilités. Avec leurs mots, Michel Houellebecq et Eric Zemmour ont pu en faire autant. Pourquoi défendre la liberté d'expression des uns quand on conteste celle des autres? [SB]

D'un côté, on plaide pour la liberté d'expression et de l'autre côté on n'a le droit de s'exprimer en mode 'politiquement correct" ! Hypocrites ! ! ! [SB]

- Cohérence /incohérence, hypocrisie... justice que marque l'apparition de termes comme «incohérence », «hypocrisie », ou les deux à la fois :

Où est la cohérence dans la France des Charlie? Il doit y avoir des libertés d"expressions plus libres que d"autres, surtout quand les amis saoudiens s"en mêlent... [SB]

C'est étrange cette incohérence qui ressemble à de l"hypocrisie ! [SB]

D'un côté, on plaide pour la liberté d'expression et de l"autre côté on n"a le droit de s"exprimer en mode "'politiquement correct »! Hypocrites ! ! ! [SB] ce serait peut être l'occasion de réfléchir sur cette notion de «liberté d'expression » brandie à tout va et parfaitement hypocrite: quand Mme Taubira se fait caricaturer en singe les mêmes ne parlent pas de liberté d'expression. [SB]

\subsection{Un traitement injuste de certaines catégories de victimes}

Je me suis initialement penchée sur toutes les formes prises par l'invocation de la règle de justice, que ce soit pour en réclamer l'application ou pour en dénoncer le nonrespect, et quel qu'en soit l'objet spécifique (Doury 2017a, b). On s'arrêtera spécifiquement ici sur la dénonciation du non-respect de la règle de justice au regard du traitement des victimes, telle qu'elle est apparue dans de nombreux commentaires postés sur Internet, suggérant que les victimes insuffisamment reconnues seraient en quelque sorte des « victimes au carré ».

\subsubsection{Des sous-catégories de victimes}

Les messages au cœur de cette étude posent que toutes les victimes ne font pas l'objet d'un même traitement, mais que la mort ou les souffrances de certaines d'entre elles sont considérées indûment comme "plus importantes » que d'autres, en fonction de différents critères qui construisent des sous-catégories souterraines justifiant implicitement des distinctions iniques - et c'est, précisément, ce caractère souterrain qui détermine l'injustice, qui, rappelle Perelman (1963), consiste dans le fait de violer les règles de justice concrète qui ont été explicitement admises comme fondant le jugement.

Argumentation et Analyse du Discours, 23 | 2019 


\section{- Selon leur religion}

\section{traitement des victimes des attentats s'appuie sur la dénonciation d'une discrimination religieuse, la gravité des préjudices subis étant alignée sur leurs préférences cultuelles. On peut s'y attendre, sur le Salon Beige ${ }^{8}$, on déplore que les réactions soient moindres lorsque ce sont des chrétiens qui sont touchés : \\ il parait que Mgr Vingt-Trois hésite toujours à faire sonner le glas pour tous ces chrétiens égorgés et toutes ces femmes violées par les tenants de l'islam.... Ils ne doivent pas être aussi « bien » que Charlie... [SB] \\ Le MASSACRE DES POPULATIONS dans tous les pays où sévit l'ISLAM n'a pas donné lieu à un tel élan de compassion et de (soit-disant) unité du pays et du gouvernement! \\ Mais il est vrai, ce sont des CHRÉTIENS, qui sont massacrés ! Ce n'est pas grave. [SB]}

façon caractéristique, la dénonciation d'un "deux poids, deux mesures" dans le

L'évaluation des réactions au sort des victimes juives est contrastée. Il arrive que certains commentaires déplorent une minimisation médiatique et politique de la gravité des actes commis contre elles, comme dans ce micro-trottoir entendu sur France Info le 9 janvier 2016, dans un sujet consacré à la commémoration de l'attentat contre l'hypercasher de la porte de Vincennes :

Josiane, 70 ans, prend les choses avec moins de sourire, surtout depuis les attentats de Toulouse il y a cinq ans :

«Une balle dans la tête d'un enfant, il y a même pas eu de manifestation en France ; les gens se sont réveillés, pour Charlie; y aurait eu que l'hyper casher, ils auraient pas réagi, aussi !»

Josiane parle d'Israel comme d'un point de chute au cas où.

Le « deux poids, deux mesures » visé par Josiane pointe ici vers la dénonciation du fait qu'en France, on considérerait que la violence est moins grave lorsqu'elle est dirigée contre des Juifs; et l'argument qui soutient cette dénonciation est la mise en parallèle, d'une part, des réactions à l'attentat contre une école juive en mars 2012 à Toulouse, associées à l'hypothétique absence de réactions dans le cas (contre-factuel) où seul l'hyper-casher aurait été ciblé en janvier 2015, et d'autre part, la mobilisation massive qui a effectivement eu lieu pour dénoncer l'attentat contre Charlie Hebdo. C'est donc ici, selon Josiane, la communauté juive qui serait injustement traitée.

Mais sur les sites considérés, il arrive bien plus fréquemment que les internautes considèrent que les réactions émotionnelles véhiculées par les médias sont exacerbées dès que ce sont des juifs qui sont visés. De nombreux messages sur le Salon beige dénoncent ainsi les mesures de protection prises devant les synagogues à la suite des attentats, mises en contraste avec la faiblesse ou l'absence de protection des églises9.

\section{- Selon leur position de pouvoir}

41 Certains messages posent que l'importance accordée par les médias et les politiques aux attentats commis dans le monde témoigne de leur position sur une échelle de pouvoir politico-économique. Ainsi, seraient considérées comme des "sous-victimes » les cibles de violences commises dans les pays du tiers-monde ou dans les pays à majorité musulmane, contrairement aux victimes occidentales chrétiennes. 


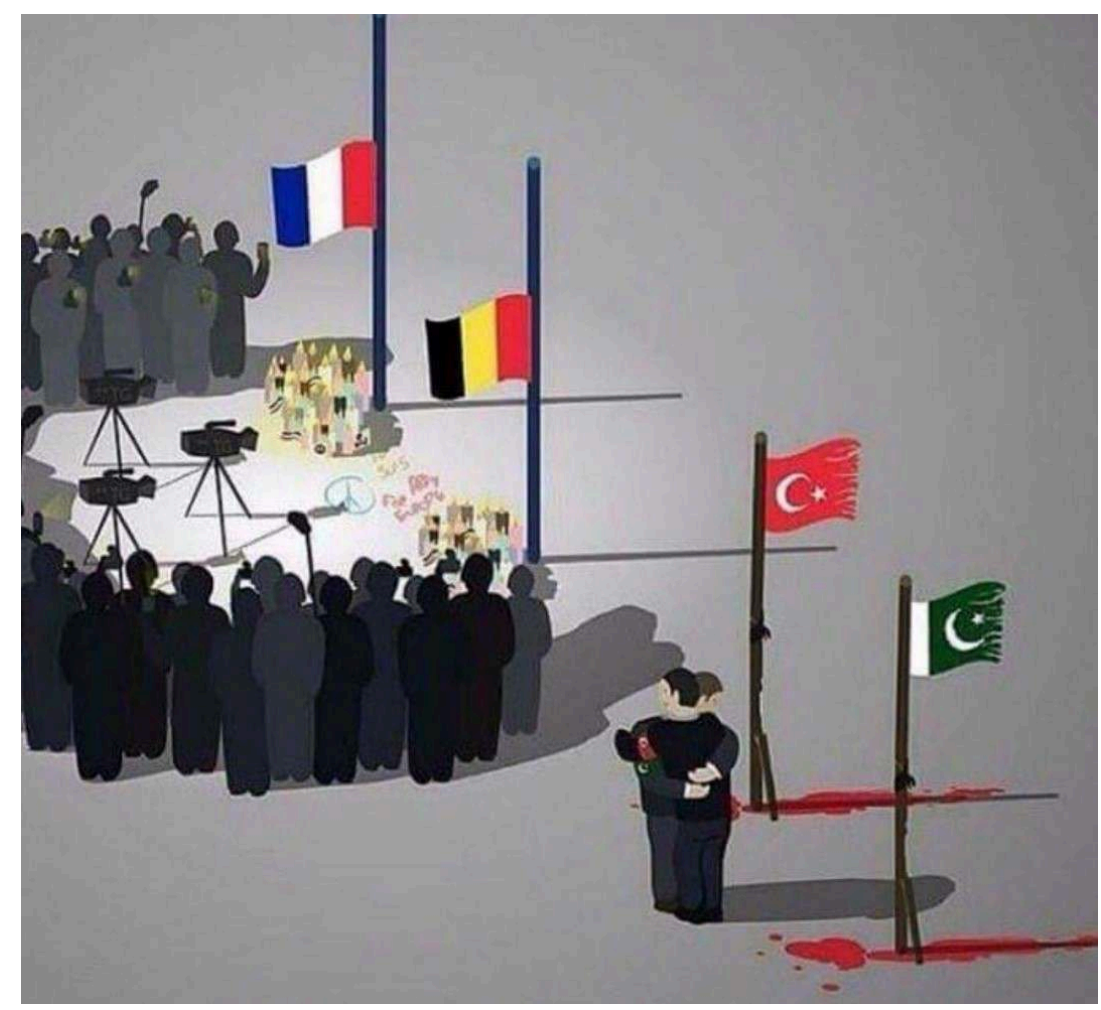

Dessin publié en juin 2016 sur le site « Les Répliques » suicide commis à l'aéroport d'Istanbul, attentat qui a fait 36 morts et 150 blessés, dénonce le fait qu'un même traitement médiatico-politique ne soit pas appliqué à l'ensemble de la catégorie "pays touchés par le terrorisme", et que les médias considèrent en fait que cette catégorie générale doit être subdivisée en sous-catégories (les pays occidentaux de tradition chrétienne, représentés ici par la France et la Belgique, et les pays à majorité musulmane, représentés par la Turquie et le Pakistan), chacune de ces sous-catégories justifiant d'un traitement propre.

Cuit, publié comme commentaire d'un article datant du 11 janvier 2015 et intitulé « 2000 tués par Boko Haram : le président de la conférence des évêques du Nigeria reproche son silence et son inaction à l'Occident ", illustre la dénonciation d'une même logique de discrimination des victimes, et met en regard les victimes "gaucho-anarchistes » de Charlie Hebdo pleurées par Hollande, Sarkozy, Fabius, BH Lévy, responsables qui auraient du sang sur les mains, ayant armé Boko Haram, et les victimes des exactions de ce mouvement terroriste au sort desquelles ces responsables seraient indifférents au motif qu'elles seraient « des noirs vivant en Afrique, pas des juifs, des chrétiens, des gaucho-anarchistes » :

Les responsables qui pleurent Charlie sont ceux qui ont armé Boko Haram, Etat Islamique, etc. : Hollande, Sarkozy, Fabius, BH Lévy...

Alors là, ils ne pleurent pas : les victimes sont des noirs vivant en Afrique, pas des juifs, des chrétiens, pas des gaucho-anarchistes. [SB]

La même critique s'exerce à l'échelle nationale: des victimes issues des fractions «dominées » de la population feraient l'objet d'une compassion bien timide au regard de victimes issues des « élites » : 
OHHH ils ont tiré sur Charlie HEBDO ohh ils ont fait 12 morts, Ne se leurrons pas, une Famille ARABE, MUSULMANE, BLACK.. n'aurait pas attiré autant d'attention !!! EMILIE je suis de tout coeur avec toi ! ! [FB]

L'importance accordée aux victimes s'organiserait également sur une échelle de notoriété, les réactions médiatiques étant massives lorsque les victimes sont connues, et moindre lorsqu'il s'agit de quidams :

on recole skon scherche au tour des femen bientot, jmenballe les couilles dvotre charlie de merde, y'a des personnes qui se fon tuer tou les jour dans le monde et en parle pa , eux parce quil sont connu qui fo les soutenir Zeubii [FB]

Merci M Meltoisan de rappeler qu'un agent chargé du nettoyage fait partie des victimes.

Qu'il repose dans la paix du Seigneur.

En dehors des signatures de Charlie et des policiers, les autres peuvent agoniser en paix et surtout dans le silence.

Faudrait voir à pas déranger les bobos. [SB]

De façon similaire, sont dénoncées également l'absence de réaction massive lors $d u$ décès de soldats français à l'étranger, ou l'insuffisance des réactions lors de la mort de policiers, au regard de l'émotion soulevée par l'attentat contre Charlie Hebdo.

\section{- Selon leurs préférences politiques}

4 Enfin, certains internautes dénoncent le fait que la mort ou la souffrance de certaines victimes soit considérée comme plus "grave » que les tourments d'autres victimes en raison de leurs préférences politiques : les «bonnes » victimes seraient les victimes de gauche, les victimes de droite étant des épiphénomènes.

"Selon que vous serez de gauche..."

Quelque chose me dit que si c'était la rédaction de Valeurs Actuelles qui avait été

décimée, il n'y aurait pas eu la mobilisation que l'on constate ce soir... [SB]

Si un attentat avait été fait contre le journal Rivarol, la bande à Charlie-Hebdo s'en

gausserait avec la quasi totalité des politichiens goche/droate, et jubilerait aussi si les victimes étaient fn ....

Mais c'est Charlie-hebdo le lèche-babouche pro-immigration qui est victime de la haine terroriste muzz et là on en fait tout un plat ..... [SB]

La question que je me pose est celle-ci : «Si ce drame avait eu lieu dans les locaux de "Famille Chrétienne" (par exemple). Quelle aurait été la réaction des médias, du gouvernement? Aurait-on sonné le glas ? .... [SB]

La caractéristique de ces exemples de dénonciation d'un «deux poids, deux mesures » est qu'ils sont construits non sur des différences de traitement avérées, mais sur des injustices hypothétiques : l'émotion suscitée dans les médias et la classe politique par l'attentat contre Charlie Hebdo est mise en balance avec d'hypothétiques attentats contre Valeurs actuelles, Rivarol ou Famille chrétienne, ce parallèle entre les victimes étant doublé d'un parallèle entre les réactions, avérées dans le cas de Charlie Hebdo ( «la mobilisation que l'on constate ce soir", "on en fait tout un plat», on en fait "des héros » voire « des martyrs »), fantasmées dans les trois autres cas (« il n’y aurait pas eu [de] mobilisation », on «s'en gausserait », on « jubilerait », « [on n'aurait pas] sonné le glas »). Le sentiment de ne pouvoir être reconnus socialement comme victimes en raison d'un positionnement politique momentanément réprouvé rappelle singulièrement les revendications des "épurés » qui, à la suite de la deuxième guerre mondiale, ont cherché à obtenir «l'amnistie pour tous ceux qui ne furent pas des traîtres authentiques et des criminels relevant du droit commun ", et la réhabilitation 
de nombre de ceux qui avaient été condamnés pour collaboration, "accusant l'Etat français d'exactions et de meurtres"; ce mouvement, mené essentiellement par le Comité Français pour la défense des droits de l'homme (organisation d'extrême droite de défense des épurés), a défendu plus de 10000 dossiers auprès des tribunaux afin d'obtenir réparation pour des victimes que l'issue de la guerre avait rendues « illégitimes », pour reprendre les termes de Baudinière (2008).

\section{Règle de justice, dénonciation d'un « deux poids deux mesures », rhétorique du ressentiment}

La caractéristique des dénonciations d'un "deux poids, deux mesures" dans le traitement politico-médiatique des victimes des attentats de janvier 2015 (entre elles, ou au regard d'autres victimes) est que, contrairement à ce qu'on aurait pu attendre, elles ne servent que rarement des argumentations a pari visant à revendiquer un traitement équitable de toutes les victimes par application de la forme générale de la règle de justice, selon laquelle, on l'a vu, « les êtres d'une même catégorie essentielle doivent être traités de la même façon » - la catégorie essentielle pertinente étant ici «les victimes d'attentats» (ou plus largement, «les victimes d'actions violentes»). Ce qu'on trouve massivement, ce sont des séquences dénonçant le non-respect de la règle de justice, sans le plus souvent qu'aucune conclusion explicite ne soit visée au-delà de cette dénonciation - on a même le sentiment que la dénonciation d'un « deux poids, deux mesures " a un statut de conclusion, que viendrait étayer l'évocation de cas particuliers: elle entre dans une séquence argumentative comme proposition ( «le monde n'est pas juste ») dont il s'agit de démontrer le bien-fondé, au terme d'un raisonnement qui s'appuie sur la nécessité de respecter la règle de justice comme prémisse majeure (généralement implicite), et une prémisse mineure relatant un exemple concret de non-respect de cette règle.

\subsection{Les appels à la règle de justice à composante fictionnelle}

Particulièrement pertinente de ce point de vue est la forme contre-factuelle prise par nombre de ces séquences ${ }^{11}$ : «si les victimes avaient appartenu à telle ou telle souscatégorie, alors.... » : dans ces " expériences de pensée », l'injustice n'est pas constatée sur la base de faits, mais posée d'emblée, et étayée par l'évocation d'une situation fictive mais présentée comme allant de soi. Le point de départ semble en être le sentiment d'appartenir à une catégorie de la population " maltraitée " politiquement et médiatiquement - ou du moins, de s'en sentir solidaire. Ce sentiment est argumenté par la mise en contraste du traitement réservé aux victimes de l'attentat contre Charlie Hebdo avec des situations qui, pour être fictives, n'en sont pas pour autant dépourvues de force d'étayage. Angenot met le doigt sur « le statut cognitif et ontologique bizarre " de tels raisonnements, «bizarre en ceci que "Si j'avais pris cet itinéraire hier, j'aurais été bloqué par la manif" est contraire aux faits, fictif, mais néanmoins plus vrai (moins faux?) que “...je n'aurais pas été bloqué par la manif”, s'il est admis que des manifestants ont bloqué le carrefour toute l'après-midi » (2008: 229). Et quel que soit son positionnement politique et ce qu'on a ressenti face à l'attentat contre Charlie Hebdo, on peut difficilement disqualifier comme improbable la situation fictive avancée au service de la dénonciation d'un « deux poids, deux mesures » dans le traitement des 
victimes : j'imagine en effet difficilement qu'un attentat commis contre Valeurs actuelles ou Rivarol aurait suscité une telle vague de réactions, tant à l'échelle nationale qu'à l'échelle internationale.

\subsection{Dénonciation du non-respect de la règle de justice dans le traitement des victimes et rhétorique du ressentiment}

51 Si la thèse d'un traitement différencié des victimes était elle-même mise au service d'une demande de réparation à l'égard des victimes injustement minorées, ou de l'exigence d'un engagement à rétablir à l'avenir un traitement équitable, la dénonciation d'un "deux poids, deux mesures" pourrait être considérée comme témoignant d'une démarche constructive, visant l'établissement d'une société plus juste.

Mais en l'état, elle semble moins orientée vers la mise en œuvre d'actions de réparation spécifiques que vers le renforcement d'une forme de ce que Angenot appelle "l'idéologie du ressentiment », et qu'il décrit ainsi :

Je qualifie de ressentiment un mode de production du sens, des valeurs, d'images identitaires, d'idées morales, politiques et civiques qui repose sur quelques présupposés et qui vise à un renversement des valeurs dominantes - Umwertung der Werte - et à l'absolutisation de valeurs "autres", inverses de celles qui prédominent, valeurs censées propres à un groupe dépossédé et revendicateur. Cette rhétorique va servir deux fins concomitantes: démontrer la situation présente comme injustice totale, persuader de l'inversion des valeurs qui se trouve à son principe et expliquer la condition inférieure des siens en renvoyant ad alteram partem tous les échecs essuyés. Seconde finalité : valoriser les positions victimales et le mode d'être du dominé ; dévaloriser les valeurs que chérit le dominant et qui vous sont inaccessibles en les montrant à la fois (cette simultanéité est déjà paralogique) comme dédaignables, chimériques, arbitraires, ignobles, usurpées et causatrices de préjudice » (Angenot $2008: 343$ ).

Il s'agit pour Angenot d'une idiosyncrasie argumentative, qui constitue le terreau sur lequel prospèrent les théories conspiratoires, qui s'exprime sur le mode du «ressassement collectif de griefs et de rancunes» (2007: § 29), et qu'il est parfois difficile de distinguer clairement d'un esprit de révolte authentique et de légitimes aspirations à la justice, sous l'apparence desquels il se présente souvent. Mais l'idéal d'égalité au nom duquel les «deux poids, deux mesures» sont dénoncés est sérieusement mis à mal par l'observation que bien souvent les argumentations a pari, demandant à ce que tout le monde soit traité de façon similaire, se transforment en fait en argumentations a fortiori : la catégorie dont je considère qu'elle est injustement traitée ne devrait pas seulement être traitée comme la catégorie favorisée ; elle devrait en fait être « mieux » traitée, parce qu'elle est plus méritante, plus « innocente », parce qu'elle a plus souffert, etc... On passe donc facilement, de la revendication d'une égalité de traitement, à la revendication d'un traitement de faveur accordé au groupe dont on se réclame. Cette observation rejoint une fois encore une caractéristique de la rhétorique du ressentiment qui, souligne Angenot, constitue un puissant facteur de cohésion identitaire de certains groupes sociaux :

Le ressentiment, qui recrée une solidarité entre pairs rancuniers et victimisés et valorise le repli communautaire, apparaît comme un moyen de réactiver à peu de frais de la chaleur; de la communion dans l'irrationnel chaleureux, alors qu'on se trouve confronté à des mécanismes de développement sociaux et internationaux 
anonymes et froids, des «monstres froids » incontrôlables, lesquels ne permettent justement pas de tactique ni de réussites collectives. Je vois dans les retours actuels $\mathrm{du}$ ressentiment quelque chose qui vient colmater les trous, boucher les vides dans une conjoncture qui dépossède les esprits de tout projet d'espérance commune et rend suspicieux à l'égard de la démocratie et de l'Etat de droit. [2007 : § 38] des acteurs sociaux affirment que le préjudice initialement subi se double d'un préjudice secondaire causé par une minoration de la souffrance, élevant le statut des victimes à celui de victimes au carré, statut susceptible d'«éveiller l'attention publique » et de leur permettre de «se tailler une place sur l'échiquier sociopolitique » (Benbassa 2006: 586).

\section{Conclusion}

L'appel à la règle de justice peut être mis au service d'une argumentation orientée vers la restauration d'une forme de justice sociale. C'est le cas lorsqu'il constitue la prémisse d'une argumentation a pari ; il est alors au cœur de ce que Juliette Rennes $(2007,2013)$ appelle des controverses d'égalité, controverses dans lesquelles des revendications contradictoires à l'égalité des droits sont confrontées à des dispositifs de résistance pratiques et discursifs spécifiques dans des régimes soumis à des normes égalitaires et libérales (2013: 96). Dans de telles controverses (dont le débat qui a abouti à l'ouverture du mariage aux couples de même sexe en France en 2013, ou tous les débats qui, historiquement, ont accompagné ou permis l'extension des droits des femmes), la dénonciation d'une transgression de la règle de justice vise à sa restauration.

Dans les exemples traités ici, il s'agit de dénoncer le fait que les médias et la sphère politique, nationale ou internationale, appliquent une règle de justice contestable, en ce qu'elle repose sur la construction et la hiérarchisation tacite de sous-catégories de victimes; ces sous-catégories ordonnées seraient au principe d'une application proportionnelle de la compassion, selon des déclinaisons spécifiques de la formule générale : "à chacun selon sa religion ", «à chacun selon sa distance au pouvoir ", "à chacun selon ses préférences politiques "... Il ne s'agit pas de nier que de telles souscatégorisations puissent effectivement être à l'œuvre dans la médiatisation des différents attentats qui frappent depuis des années différentes cibles, et dont la couverture varie spectaculairement. Les travaux initiés depuis les années 60 sur la notion de «news values » ont mis au jour bon nombre des logiques de filtrage à l'œuvre dans les médias, et qui gouvernent le processus de médiatisation qui fait passer un « event» au statut de "news» (Galtung et Ruge 1965, Östgaard 1965). Certaines des logiques de discrimination dénoncées dans les exemples analysés ici peuvent ainsi être interprétées comme des variantes du principe de proximité, qui veut que les médias favorisent ce qui nous est proche (géographiquement, culturellement, et, dans les messages postés après les attentats, religieusement ou politiquement). La critique ainsi ouverte est saine si elle ouvre à une réflexion sur ce que serait un traitement différencié (puisque chaque conjoncture est différente, chaque attentat est particulier, chaque victime est unique) mais équitable (aucune mort, aucune souffrance, directe ou indirecte, causée par de tels actes, ne devrait être minorée). Elle est en revanche problématique lorsqu'elle entre essentiellement au service d'une rhétorique du ressentiment, qui génère une forme de repli sur soi et engendre, soutient, nourrit des idéologies nationalistes et identitaires (Angenot 2007 : § 29).

Argumentation et Analyse du Discours, 23 | 2019 
Une précision enfin : je n'affirme en aucun cas que les procédés décrits ici constituent de nouvelles formes d'argumentation, qui auraient émergé après les événements de janvier 2015; mais il m'a semblé que la logique qui les sous-tend les rend particulièrement propres à porter l'expression de certaines des tensions qui traversent la société française actuellement, et reflètent une sorte de tropisme vers la fragmentation que l'idéologie du ressentiment tend à rendre pérenne. Dans le cas des commentaires postés suite à l'attentat contre Charlie Hebdo, ces procédés suggèrent une sorte de repli communautaire centré sur des identités essentiellement religieuses (les musulmans /les catholiques), ou politiques (les " gauchos »/les gens de droite); mais le mouvement des Gilets Jaunes qui constitue l'actualité politique française de ces derniers mois ${ }^{13}$ fait retentir des dénonciations structurellement similaires, témoignant davantage de fractures sociales ou économiques, dénonçant des «deux poids, deux mesures » liés au capital économique ou à la proximité du pouvoir.

\section{BIBLIOGRAPHIE}

Albertini, Dominique \& David Doucet. 2016. La fachosphère. Comment l'extrême droite remporte la bataille du net (Paris : Flammarion).

Angenot, Marc. 2007. « Nouvelles figures de la rhétorique : la logique du ressentiment », Questions de communication $12,57-75$

Angenot, Marc. 2008. Dialogues de sourds. Traité de rhétorique antilogique (Paris : Mille et une nuits).

Angenot, Marc. 2010. «The Ideologies of Resentment » (Traduit par B. Barsky). Ameriquests, Nashville TN, e-journal published by Vanderbilt University. http://

ejournals.library.vanderbilt.edu.

Badouard, Romain. 2016. «"Je ne suis pas Charlie". Pluralité des prises de parole sur le web et les réseaux sociaux ", Lefébure, Pierre \& Claire Sécail (éds). Le défi Charlie. Les médias à l'épreuve des attentats (Paris : Lemieux éditeur), 187-219.

Baudinière, Caroline. 2008. « Une mobilisation de victimes illégitimes. Quand les épurés français de la Seconde Guerre mondiale s'engagent à l'extrême-droite ", Raisons politiques 30-2, 21-39.

Benbassa, Esther. 2006. " La concurrence des victimes », Blanchard, Pascal \& Nicolas Bancel (éds). Cultures post-coloniale, 1961-2006 (Paris : Autrement), 102-112. Repris dans : Blanchard, Pascal, Sandrine Lemaire \& Nicolas Bancel (éds). 2008. Culture coloniale en France. De la révolution française à nos jours (Paris : CNRS Editions), 585-594

Chaumont, Jean-Michel. 2000. « Du culte des héros à la concurrence des victimes », Criminologie $33-1,167-183$

Corey, Michael, Troisi, Jordan D. \& Nicksa, Sarah C. 2015. « Tipping the Scales of Justice : The Influence of Victimization on Belief in a Just Word », Social Justice Research 28-4, 509-525

Doury, Marianne. 2017a. « L'exploitation argumentative de la règle de justice dans des réactions aux attentats de 2015 en France ». La Clé des Langues [en ligne], Lyon, ENS de LYON/DGESCO (ISSN 2107-7029). Consulté le 20/04/2018. URL : http://cle.ens-lyon.fr/plurilangues/a-trier/l- 
exploitation-argumentative-de-la-regle-de-justice-dans-des-reactions-aux-attentats-de-2015-enfrance

Doury, Marianne. 2017b. « On arguments based on the justice rule in reactions to the 2015 terrorist attacks in France ", Colloquium of the Reasoning and Argumentation Lab (ArgLab), Lisbonne Doury, Marianne. 2018. « Le marquage langagier des types d'arguments. Le cas de l'argumentation par l'absurde ». Herman, Thierry, Jérôme Jacquin \& Steve Oswald (éds). Les mots de l'argumentation (Berne : P. Lang), 27-55

van Eemeren, Frans H. et al. 2014. Handbook of Argumentation Theory (Dordrecht : Springer)

van Eemeren, Frans H. \& Rob Grootendorst. 1992. Argumentation, communication, and fallacies : a pragma-dialectical perspective (Hillsdale, $\mathrm{NJ}$ : Erlbaum)

van Eemeren, Frans H., Peter Houtlosser \& Francisca Snoeck Henkemans. 2007. Argumentative Indicators in Discourse. A Pragma-Dialectical Study (Dordrecht : Springer)

Galtung, Johan \& Ruge, Mari Holmboe. 1965. « The Structure of Foreign News », Journal of Peace Research 2-1, 64-91

Garssen, Bart. 2002. "Understanding argumentation schemes”, van Eemeren Frans H. (éd.). Advances in pragma-dialectics (Amsterdam : SicSat/Vale press), 93-104

Gombin, Joël, Bérénice Mariau \& Gaël Villeneuve. 2016. « Le web politique au lendemain des attentats de Charlie ", Lefébure, Pierre \& Claire Sécail (éds). Le défi Charlie. Les médias à l'épreuve des attentats (Paris : Lemieux éditeur), 221-256

Hafer, Carolyn L. \& Gosse, Leanne. 2011. « Predicting alternative strategies for preserving a belief in a just world : The case of repressive coping style », European Journal of Social Psychology 41, 730-739

Jacquin, Jérôme \& Raphaël Micheli. 2012. « Entre texte et interaction : propositions méthodologiques pour une approche discursive de l'argumentation en sciences du langage », SHS Web of Conferences Volume 1 ; 3e Congrès Mondial de Linguistique Française, 599-611.

Koren, Roselyne. 2011. « De la rationalité et/ou de l'irrationalité des polémiqueurs : Certitudes et incertitudes ", Semen [En ligne], 31, mis en ligne le 01 avril 2011, consulté le 02 février 2019. URL : http://journals.openedition.org/semen/9061

Koren, Roselyne. 2019. Rhétorique et Éthique : du jugement de valeur (Paris : Garnier).

Lerner, Melvin J. \& Simmons, Carolyn H. 1966. « Observer's reaction to the "innocent victim" : compassion or rejection? », Journal of Personality and Social Psychology 4-2, 203-210

Lerner, Melvin J. \& Miller, Dale T. 1978. « Just world research and the attribution process :

Looking back and ahead », Psychological Bulletin 85-5, 1030-1051

Micheli, Raphaël. 2012. « Arguing Without Trying to Persuade ? Elements for a Non-Persuasive Definition of Argumentation ", Argumentation 26, 115-126.

Östgaard, Einar. 1965. « Factors Influencing the Flow of News », Journal of Peace Research 1, 39-63

Perelman, Chaïm. 1963. The Idea of Justice and the Problem of Argument (London : Routledge \& Kegan Paul ; New York: The Humanities Press)

Perelman, Chaïm. 1990 [1945]. De la justice, paru dans la collection des Actualités Sociales, nouvelle série, Université Libre de Bruxelles, Institut de Sociologie Solvay, Bruxelles, Office de Publicité. Repris dans Perelman, Chaïm. 1990. Ethique et droit (Bruxelles : Ed. de l'université de Bruxelles), 13-86 
Perelman, Chaïm \& Lucie Olbrechts-Tyteca. 1988 [1958]. Traité de l'argumentation. La nouvelle rhétorique (Bruxelles : Editions de l'Université de Bruxelles)

Plantin, Christian. (2002). Analyse et critique du discours argumentatif », Koren, Roselyne \& Ruth Amossy (éds). Après Perelman : quelles politiques pour les nouvelles rhétoriques? (Paris : L'Harmattan), 229-263

Plantin, Christian. 2016. Dictionnaire de l'argumentation. Une introduction aux études d'argumentation (Lyon : ENS éditions)

Rennes, Juliette. 2007. Le mérite et la nature. Une controverse républicaine : l'accès des femmes aux professions de prestige, 1880-1940 (Paris : Fayard)

Rennes, Juliette. 2013. « Analysing controversy. The contributions of argumentation study to political science ", Bonnafous, Simone \& Malika Temmar (éds). Discourse analysis in human and social sciences (Bern : P. Lang), 93-112

Soudan, Coralie \& Bernard Gangloff. 2012. «La croyance au monde juste comme facteur explicatif de diverses réactions à des injustices professionnelles ", Psychologie du travail et des organisations $17,171-185$

Todorov, Tzvetan. 1995. « Du culte de la différence à la sacralisation de la victime », Esprit 212, 90-102

\section{NOTES}

1. On aura reconnu ici la tripartition proposée par l'école pragma-dialectique, qui distingue les argumentations comparatives (reposant sur l'existence d'une relation de ressemblance entre la prémisse et la conclusion), les argumentations causales et les argumentations symptomatiques, qui recouvrent les cas où un locuteur cherche à convaincre son interlocuteur de son point de vue en suggérant qu'une chose est symptomatique d'une autre chose - autrement dit, l'acceptabilité des prémisses est transférée à la conclusion sur la base d'une relation de concomitance entre ce qui est asserté dans la prémisse, et ce qui est asserté dans la conclusion (van Eemeren \& Grootendorst 1992).

2. Un autre intérêt de la notion de type d'argument réside dans les rapports qu'elle entretient avec la critique de l'argumentation ; voir par exemple Doury $2018: 37$.

3. On trouvera, dans le dernier chapitre de Koren (2019), une restitution attentive de cette réflexion.

4. Koren (2011) propose de son côté l'analyse d'une polémique parue en 2006 dans Libération entre Shmuel Trigano, Bruno Stevens et Michel Tubiana, après la parution d'un article de Trigano dénonçant la différence de traitement médiatique entre les victimes des conflits militaires dans le monde et «l'occultation des violences subies par les civils en Israël » - Stevens et Tubiana lui reprochant en retour sa partialité en faveur d'Israël.

5. La source des exemples est indiquée après chacun entre crochets; $\mathrm{SB}=$ le Salon Beige; FB = page officielle de Charlie Hebdo sur Facebook.

6. Ce message fait référence à un album du chanteur populaire français Michel Sardou, album intitulé "Selon que vous serez, etc., etc. » sorti en 1994, et comportant une chanson éponyme, qui commence ainsi : "Je pense au jeune homme imprudent /Qui prend entre six mois, deux ans /Pour un désordre assez minable /Et ceux que la Loi n'atteint pas /On a devant soi la Justice /Et l'apparence de la justice /La nuance est indéfinissable /Ce qui est pris ne se rend pas /Selon que vous serez puissant ou misérable, etc. etc. » 
7. On trouve aussi des variantes verbo-visuelles de la dénonciation du non-respect de la règle de justice, qui reposent sur des constructions visuelles de contraste. Typiquement, elles proposent, côte à côte ou l'une sous l'autre, une visualisation de deux situations (l'une par exemple incarnant l'islam, l'autre, le christianisme), visualisation doublée d'un message verbal spécifiant la différence de traitement des deux religions ([SB, https://www.lesalonbeige.fr/libertedexpression-cest-du-cinema/]).

8. Qui se présente, rappelons-le, comme un site de « catholiques laïcs".

9. Plus encore que pour une hiérarchisation des victimes, la ligne de partage entre religions est mise sur le devant de la scène dans les messages portant sur une défense de la liberté d'expression « à deux vitesses » : sur les Juifs, « on n'aurait rien le droit de dire ", alors qu'on pourrait salir les musulmans sans retenue; les chrétiens seraient tournés en dérision sans que personne ne s'en émeuve alors que l'Islam serait « intouchable »; etc.

10. https://www.lesrepliques.com/

11. Les raisonnements contrefactuels sont des raisonnements «qui travaillent sur des mondes possibles ou raisonnent sur le monde empirique à partir de mondes alternatifs, à partir d'imaginations contraires à l'empirie » (Angenot $2008: 227$ ).

12. La "mise en concurrence des victimes" qui transparaît dans les données analysées ici se distingue nettement du phénomène décrit notamment par Benbassa (2006), Chaumont (2000) ou Todorov (1995) par le fait qu'elle ne s'inscrit pas dans le prolongement d'une histoire victimaire revendiquée, mais se déploie fondamentalement en synchronie, au-delà de toute légitimation mémorielle. Elle s'en distingue encore par le fait que le statut de « victime au carré », de « supervictime ", n'est souvent pas revendiquée par les locuteurs pour eux-mêmes, mais pour un tiers, membre d'un groupe social auquel le locuteur lui-même n'appartient pas (les " petites gens », les policiers, nos soldats à l'étranger, les victimes des attentats en Afrique...).

13. Le présent article a été rédigé en février 2019.

\section{RÉSUMÉS}

Parmi les réactions qui ont suivi les attaques terroristes islamistes qui ont eu lieu en 2015 sur le sol français, de nombreux commentaires dénoncent ce qu'ils considèrent comme un traitement inéquitable des victimes. Les victimes en question sont parfois précisément celles des attentats ('on fait plus de cas de la mort des dessinateurs de Charlie Hebdo que de celle de l'agent de maintenance'), parfois les victimes des attentats de 2015 sur le sol français au regard des victimes d'autres attentats commis dans d'autres sphères géo-politiques ('on n'en a pas fait autant pour les 29 morts de la fusillade de Sousse'); il s'agit parfois enfin d'établir un parallèle entre le traitement des victimes bien réelles avec le traitement imaginé des victimes d'un attentat fictif ('si les victimes avaient travaillé pour Valeurs actuelles, on n'en aurait pas fait tout un foin'). Partant des réflexions de Perelman sur la règle de justice, l'article examine ces dénonciations d'un « deux poids, deux mesures» dans le traitement des victimes, qui font des victimes moins reconnues, ou moins médiatisées, des victimes «au carré ». Il montre que cette règle peut être mobilisée dans des constructions argumentatives visant des conclusions différentes: dénonciation d'une injustice/ rétablissement de la justice. Il met au jour la façon dont cette "élévation au carré » de certaines victimes constitue parfois le symptôme d'une logique du ressentiment, révélatrice de tensions et de tendances au repli identitaire identifiables dans la société française actuelle. 
Among the reactions that followed the Islamic terrorist attacks of 2015 in France, many comments denounced what they considered an unfair treatment of the victims. The latter are sometimes the victims of the same terrorist attack ("one makes more case of the death of Charlie Hebdo's cartoonists than of that of the maintenance agent"); sometimes a parallel is drawn between the victims of the 2015 attack in Paris and those of other attacks in other geopolitical spheres ('The media did not care that much for the 29 victims of the Sousse shooting'); sometimes the parallel is between the treatment of real victims and the imagined treatment of victims of a fictitious attack ("if the victims had worked for Valeurs actuelles, the media would not have made such a big deal of it"). Based on Perelman's reflections on the rule of justice, the paper examines such denunciations of a "double standard" which make less recognized, or less publicized, victims, "squared" victims. It shows that the rule of justice can be used in argumentative constructions oriented to different conclusions: denunciation of an injustice/ restoration of justice. It reveals how this "squaring" of some victims is sometimes symptomatic of a logic of resentment, revealing tensions and i tendencies to identitarian closure that can be identified in today's French society.

\section{INDEX}

Mots-clés : argument a pari, deux poids deux mesures, règle de justice, rhétorique du ressentiment, victimes

Keywords : a pari argument, double standard, rhetoric of resentment, rule of justice, victims

\section{AUTEUR}

\section{MARIANNE DOURY}

Université de Paris, EDA 